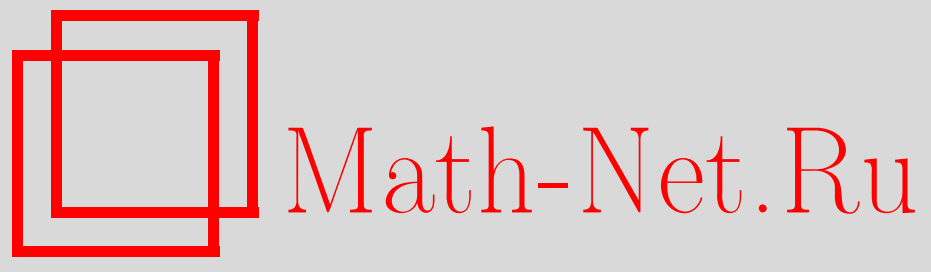

Г. Г. Геворкян, Теоремы единственности для рядов по системе Франклина, Матем. заметки, 2015, том 98, выпуск $5,786-789$

DOI: https://doi.org/10.4213/mzm10898

Использование Общероссийского математического портала Math-Net.Ru подразумевает, что вы прочитали и согласны с пользовательским соглашением http://www . mathnet.ru/rus/agreement

Параметры загрузки:

IP : 3.85 .183 .62

26 апреля 2023 г., 15:30:00

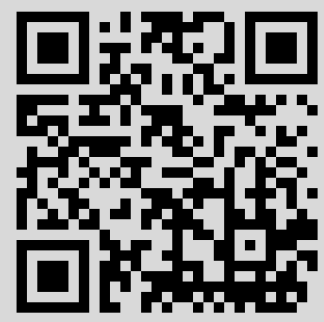




\section{Теоремы единственности для рядов по системе Франклина}

\section{Г. Г. Геворкян}

В 1928 г. Франклин [1] построил первый пример полного ортонормированного базиса в $C[0 ; 1]$. Эта система состоит из кусочно линейных непрерывных функций. Систематическое изучение системы Франклина началось с работ Чисельского [2], [3]. Далее эта система оказалась очень полезной при решении разных задач. В работе [4] сделан подробный обзор исследований рядов по системе Франклина.

В теории тригонометрических рядов широко известна теорема единственности Кантора (см. [5; с. 191]).

Теорема Кантора. Если тригонометрический ряд

$$
\frac{a_{0}}{2}+\sum_{n=1}^{\infty}\left(a_{n} \cos n x+b_{n} \sin n x\right)
$$

всюду сходится к нулю, то все коэффиииенты этого ряда равны нулю.

Эта теорема послужила отправным пунктом для развития теории единственности тригонометрических рядов. Здесь напомним только теорему Валле-Пуссена (см. [5; с. 789])

ТЕОРема ВАЛЛЕ-ПуССена. Если тригонометрический ряд (1) всюду, кроме бытъ может, некоторого счетного множества сходится $к$ всюду конечной интегрируемой функиии, то он является рядом Фуръе этой функиии.

Однако, до сих пор некоторые фундаментальные проблемы в этой области остаются открытыми. В частности, не решена следующая проблема Ульянова (см. [6; с. 24]): Следует ли из всюду сходимости к нулю некоторой подпоследовательности частичных сумм тригонометрического ряда, с ограниченными коэффициентами, что все коэффициенты этого ряда равны нулю?

С работ [7]-[9] начались исследования вопросов единственности рядов по системам Хаара и Уолша. В работах [7], [8] была доказана теорема типа Кантора для рядов Хаара. Фабер [11] показал, что даже одноточечное множество $\{1 / 2\}$ не является множеством единственности для рядов Хаара, т.е. существует нетривиальный ряд по системе Хаара, который всюду вне этого множества сходится к нулю. Оказалось (см., например, [10]), что для рядов Хаара любое одноточечное множество не является множеством единственности. В работе [10] доказаны также аналоги теоремы Валле-Пуссена для рядов по системам Хаара и Уолша, коэффициенты которых удовлетворяют некоторым необходимым условиям.

Вопросы единственности для рядов по системе Франклина менее изучены. До настоящей статьи не был известен аналог теоремы Кантора для системы Франклина. Для формулировки ранее полученных и новых результатов в этом направлении напомним некоторые определения.

Пусть $n=2^{\mu}+\nu$, где $\mu \geqslant 0,1 \leqslant \nu \leqslant 2^{\mu}$. Обозначим

$$
s_{n, i}= \begin{cases}\frac{i}{2^{\mu+1}}, & \text { для } 0 \leqslant i \leqslant 2 \nu \\ \frac{i-\nu}{2^{\mu}}, & \text { для } 2 \nu<i \leqslant n .\end{cases}
$$

Через $S_{n}$ обозначим пространство функций, непрерывных на $[0 ; 1]$ и кусочно линейных с узлами $\left\{s_{n, i}\right\}_{i=0}^{n}$, т.е. $f \in S_{n}$, если $f \in C[0 ; 1]$, и $f$ линейна на каждом отрезке $\left[s_{n, i-1} ; s_{n, i}\right]$,

Данная работа была проделана при финансовой поддержке гранта 10-3/1-41 ГКОМОН РА.

DOI: $10.4213 / \mathrm{mzm} 10898$ 
$i=1,2, \ldots, n$. Ясно, что $\operatorname{dim} S_{n}=n+1$ и множество $\left\{s_{n, i}\right\}_{i=0}^{n}$ получается добавлением точки $s_{n, 2 \nu-1}$ к множеству $\left\{s_{n-1, i}\right\}_{i=0}^{n-1}$. Поэтому, существует единственная, с точностью до знака, функция $f_{n} \in S_{n}$, которая ортогональна $S_{n-1}$ и $\left\|f_{n}\right\|_{2}=1$. Полагая

$$
f_{0}(x)=1, \quad f_{1}(x)=\sqrt{3}(2 x-1), \quad x \in[0 ; 1],
$$

получим ортонормированную систему $\left\{f_{n}(x)\right\}_{n=0}^{\infty}$, которая другим эквивалентным образом определена Франклином [1].

Наверно, первым утверждением о единственности рядов по системе Франклина была следующая теорема, сформулированная в работе [12].

Теорема 1. Пусть ряд

$$
\sum_{n=0}^{\infty} a_{n} f_{n}(x)
$$

с коэффичиентами

$$
a_{n}=o(\sqrt{n})
$$

по мере сходится $к$ некоторой ограниченной функции $f$ и всюду, кроме быть может, некоторого счетного множества, выполняется условие

$$
\sup _{N} \max _{t \in(x-1 / N ; x+1 / N)}\left|\sum_{n=0}^{N} a_{n} f_{n}(t)\right|<+\infty .
$$

Тогда ряд (2) является рядом Фурье-Франклина функиии $f$.

Аналогичные теоремы для общей системы Франклина и других систем доказаны в работе [13]. Однако, до работы [13] были доказаны другие теоремы единственности рядов по системе Франклина. В работе [14] нами доказана следующая теорема (см. [14; теорема 3]).

Теорема 2. Для того, чтобы ряд (2) был рядом Фуръе-Франклина некоторой интегрируемой функции $f$, необходимо и достаточно, чтобы этот ряд п.в. сходился $\kappa f(x)$ $u$

$$
\liminf _{\lambda \rightarrow \infty}\left(\lambda \cdot \mu\left\{x \in[0 ; 1]: \sup _{N}\left|\sum_{n=0}^{N} a_{n} f_{n}(x)\right|>\lambda\right\}\right)=0 .
$$

В работе [15] найдены формулы восстановления коэффициентов ряда (2), который п.в. сходится к некоторой функции $f$ и удовлетворяет условию (4). В той же работе рассмотрены кратные ряды по системе Франклина.

Пусть $k$ некоторое натуральное число. Рассмотрим кратные ряды Франклина

$$
\sum_{\mathbf{m} \in \mathbb{N}_{0}^{k}} a_{\mathbf{m}} f_{\mathbf{m}}(\mathbf{x})
$$

где $\mathbf{m}=\left(m_{1}, \ldots, m_{k}\right) \in \mathbb{N}_{0}^{k}$ - вектор с целочисленными неотрицательными координатами, $\mathbf{x}=\left(x_{1}, \ldots, x_{k}\right) \in[0 ; 1]^{k}$ и $f_{\mathbf{m}}(\mathbf{x})=f_{m_{1}}\left(x_{1}\right) \cdots f_{m_{k}}\left(x_{k}\right)$. Говорят, что ряд (5) сходится по прямоугольникам в точке $\mathbf{x}$, если существует предел

$$
\lim _{\mathbf{M} \rightarrow+\infty} \sum_{\mathbf{m} \leqslant \mathbf{M}} a_{\mathbf{m}} f_{\mathbf{m}}(\mathbf{x})
$$

где $\mathbf{m} \leqslant \mathbf{M}$ означает $m_{j} \leqslant M_{j}, j=1, \ldots, k$, а $\mathbf{M}=\left(M_{1}, \ldots, M_{k}\right) \rightarrow+\infty$ означает $\min _{j} M_{j} \rightarrow$ $+\infty$.

Для кратных рядов Франклина в работе [15] доказана следующая теорема (см. [15; теорема 10]). 
Теорема 3. Для того, чтобы ряд (5) бъл рядом Фуръе-Франклина некоторой функиии $f \in L \ln ^{k-1} L\left([0 ; 1]^{k}\right)$, необходимо и достаточно, чтобъ ряд (5) по прямоугольникам п.в. сходился $\kappa f(\mathbf{x})$ и выполнялось условие

$$
\liminf _{\lambda \rightarrow+\infty}\left(\lambda \cdot \mu\left\{\mathbf{x} \in[0 ; 1]^{k}: \sup _{\mathbf{M}}\left|\sum_{\mathbf{m} \leqslant \mathbf{M}} a_{\mathbf{m}} f_{\mathbf{m}}(\mathbf{x})\right|>\lambda\right\}\right)=0 .
$$

Нами доказан аналог теоремы Кантора для системы Франклина.

Теорема 4. Если ряд (2) всюду сходится $к$ нулю, то все коэффициенты этого ряда равны нулъю.

Верна более общая теорема.

ТеОрема 5. Пусть ряд (2) всюду сходится $к$ ограниченной функиии. Тогда ряд (2) является рядом Фуръе-Франклина этой функиии.

Известно, что (см. [3; теорема 3]) для ядра Дирихле системы Франклина $K_{n}(x, t)=$ $\sum_{m=0}^{n} f_{m}(x) f_{m}(t)$ выполняется неравенство

$$
\left|K_{n}(x, t)\right| \leqslant C \cdot n \cdot(2-\sqrt{3})^{n|x-t|},
$$

где $C$ - некоторая постоянная. Следовательно, для любой точки $t \in[0 ; 1]$ существует нетривиальный ряд (2), который всюду, за исключением точки $t$, сходится к нулю. Действительно, для этого достаточно положить $a_{m}=f_{m}(t)$. Однако, если на коэффициенты ряда (2) наложить условие (3), то любое счетное множество будет множеством единственности.

Теорема 6. Пусть ряд (2) с коэффициентами (3) по мере сходится к нулю и всюду кроме, быть может, некоторого счетного множества выполняется

$$
\sup _{n}\left|\sum_{m=0}^{n} a_{m} f_{m}(x)\right|<+\infty .
$$

Тогда все коэфбициенты ряда (2) равнъь нулю.

Теорема 6 дает положительный ответ на вопрос, сформулированный в [16]. Легко заметить, что из оценок ядра Дирихле системы Франклина следует, что ряд Фурье-Франклина ограниченной функции по мере сходится и во всех точках удовлетворяет условию (7). Поэтому из теоремы 6 немедленно следует

Теорема 7. Пусть ряд (2) с коэфбиииентами (3) по мере сходится к ограниченной функиии и всюду кроме, быть может, некоторого счетного множества выполняется (7). Тогда ряд (2) является рядом Фуръе-Франклина этой функиии.

Здесь отметим одну теорему Вронича [17], указывающую на важность выполнения условия (7) для всей последовательности частичных сумм ряда (2).

Теорема Вронича. Существует нетривиалъный ряд по системе Франклина (2), для которого

$$
\lim _{\nu \rightarrow \infty} \sum_{n=0}^{2^{\nu}} a_{n} f_{n}(x)=0 \quad \text { для всех } \quad x \in[0 ; 1] .
$$

Вернемся к теоремам 2 и 3 . Через $\sigma_{\nu}(\mathbf{x})$ обозначим квадратичные частные суммы ряда (5) с номерами $2^{\nu}$, т.е.

$$
\sigma_{\nu}(\mathbf{x})=\sum_{\mathbf{m}: m_{i} \leqslant 2^{\nu}} a_{\mathbf{m}} f_{\mathbf{m}}(\mathbf{x})
$$

где $\mathbf{m}=\left(m_{1}, \ldots, m_{k}\right)$. Нами доказано следующее утверждение. 
ТеОрема 8. Для того, чтобы ряд (5) бъл рядом Фуръе-Франклина некоторой функиии $f \in L\left([0 ; 1]^{k}\right)$, необходимо и достаточно, чтобы выполнялись следуюшие условия:

1) суммы $\sigma_{\nu}(\mathbf{x})$ по мере сходятся $\kappa f$;

2) $\liminf _{\lambda \rightarrow+\infty}\left(\lambda \cdot \mu\left\{\mathbf{x} \in[0 ; 1]^{k}: \sup _{\nu}\left|\sigma_{\nu}(\mathbf{x})\right|>\lambda\right\}\right)=0$.

Метод доказательства этой теоремы существенно отличается от метода доказательств теорем 2 и 3. Доказательство теоремы 3 опирается на теорему 2, и поэтому есть потеря: вместо класса интегрируемых функций в теореме 3 получается класс $L \ln ^{k-1} L$.

Теорема 8 является новой даже в одномерном случае. Во-первых, условие 2 в теореме 8 существенно слабее условия (4) теоремы 2. Во-вторых, в теореме 2 требуется сходимость ряда (2), а в теореме 8 требуется сходимость последовательности $\sum_{n \leqslant 2^{\nu}} a_{n} f_{n}(x)$. Как указывает теорема Вронича, в этом есть существенная разница.

В связи с полученными результатами возникают следующие вопросы.

Вопрос 1. Можно ли в теоремах 5 и 7 вместо ограниченности функции $f$ потребовать интегрируемость и всюду конечность этой функции?

Вопрос 2. Из теоремы 4 следует, что не существуют два разных ряда по системе Франклина, которые всюду сходятся к одной и той же функции $f$. Как можно определить коэффициенты всюду сходящегося ряда по системе Франклина по его сумме?

Вопрос 3. Пусть известна последовательность $\lambda_{q}$, которая реализует условие 2 в теореме 8 , т.е.

$$
\lim _{q \rightarrow+\infty}\left(\lambda_{q} \cdot \mu\left\{\mathbf{x} \in[0 ; 1]^{k}: \sup _{\nu}\left|\sigma_{\nu}(\mathbf{x})\right|>\lambda_{q}\right\}\right)=0 .
$$

Можно ли выразить коэффициенты $a_{\mathbf{m}}$ через последовательность $\lambda_{q}$ и функцию $f$ ? В работе [15] это сделано для одномерных рядов по системе Франклина, при условии, что выполняется (4) (см. [15; теорема 3]).

Когда ряд (2) п.в. сходится к некоторой функции $f$ и вместо (4) выполняется

$$
\lim _{\lambda \rightarrow \infty}\left(\lambda \cdot \mu\left\{x \in[0 ; 1]: \sup _{N}\left|\sum_{n=0}^{N} a_{n} f_{n}(x)\right|>\lambda\right\}\right)=0
$$

тогда (см. [15; теорема $\left.\left.3^{\prime}\right]\right)$ функция $f A$-интегрируема и ряд $(2)$ является рядом ФурьеФранклина функции $f$ в смысле $A$-интегрирования.

Вопрос 4. Пусть в $(9) \lambda_{q}=q$. Может ли $A$-интеграл восстановить коэффициенты ряда $(5)$ ?

\section{СПИСОК ЦИТИРОВАННОЙ ЛИТЕРАТУРЫ}

[1] Ph. Franklin, Math. Ann., 100 (1928), 522-529. [2] Z. Ciesielski, Studia Math., 23 (1963), 141-157. [3] Z. Ciesielski, Studia Math., 27 (1966), 289-323. [4] Z. Ciesielski, A. Kamont, Approximation Theory, DARBA, Sofia, 2002, 84-132. [5] H. К. Бари, Тригонометрические ряды, Физматгиз, М., 1961. [6] П. Л. Ульянов, УМН, 19:4 (1964), 3-92. [7] Ф. Г. Арутюнян, Докл. АН Арм. ССР, 38:3 (1964), 129-134. [8] М. Б. Петровская, Изв. АН СССР. Сер. матем., 28:4 (1964), 773-798. [9] В. А. Скворцов, Вестн. Моск. ун-та. Сер. 1. Матем., мех., 1964, № 5, 3-6. [10] Ф. Г. Арутюнян, А. А. Талалян, Изв. АН СССР. Сер. матем., 28:6 (1964), 1391-1408. [11] G. Faber, Deutsche Math. Ver., 19 (1910), 104-112. [12] Г. Г. Геворкян, Уч. зап. Ереванск. гос. ун-та. Eстеств. науки, 1986, № 2, 146-148. [13] G. G. Gevorkyan, A. Kamont, J. Contemp. Math. Anal., 44:5 (2009), 271-283. [14] Г. Г. Геворкян, Матем. заметки, 46:2 (1989), 51-58. [15] Г. Г. Геворкян, Матем. заметки, 59:4 (1996), 521-545. [16] G. G. Gevorkyan, Approximation and Probability, Banach Center Publ., 72, Polish Acad. Sci., Warszawa, 2006, 85-92. [17] Z. Wronicz, Opusc. Math. (to appear).

\section{Г. Г. Геворкян}

